\title{
Gradhiva
}

GRADHIV

Revue d'anthropologie et d'histoire des arts

4 | 2006

Le commerce des cultures

\section{Le musée Leleque et le groupe Benetton en Patagonie}

Photographies mémoire et parrainage privé

The Leleque museum and the Benetton Group in Patagonia

\section{Julio Vezub}

Traducteur : Adrien Delmas

\section{OpenEdition}

Journals

\section{Édition électronique}

URL : http://journals.openedition.org/gradhiva/612

DOI : 10.4000/gradhiva.612

ISSN : 1760-849X

Éditeur

Musée du quai Branly Jacques Chirac

\section{Édition imprimée}

Date de publication : 1 novembre 2006

Pagination : 53-69

ISBN : 2-915133-44-1

ISSN : 0764-8928

\section{Référence électronique}

Julio Vezub, « Le musée Leleque et le groupe Benetton en Patagonie », Gradhiva [En ligne], 4 | 2006, mis en ligne le 22 décembre 2009, consulté le 19 avril 2019. URL : http://journals.openedition.org/ gradhiva/612 ; DOI : 10.4000/gradhiva.612

Ce document a été généré automatiquement le 19 avril 2019

(c) musée du quai Branly 


\section{Le musée Leleque et le groupe Benetton en Patagonie}

Photographies mémoire et parrainage privé

The Leleque museum and the Benetton Group in Patagonia

\section{Julio Vezub}

Traduction : Adrien Delmas

Manifestation indigène lors de l'inauguration du musée Leleque, 12 mai 2000.

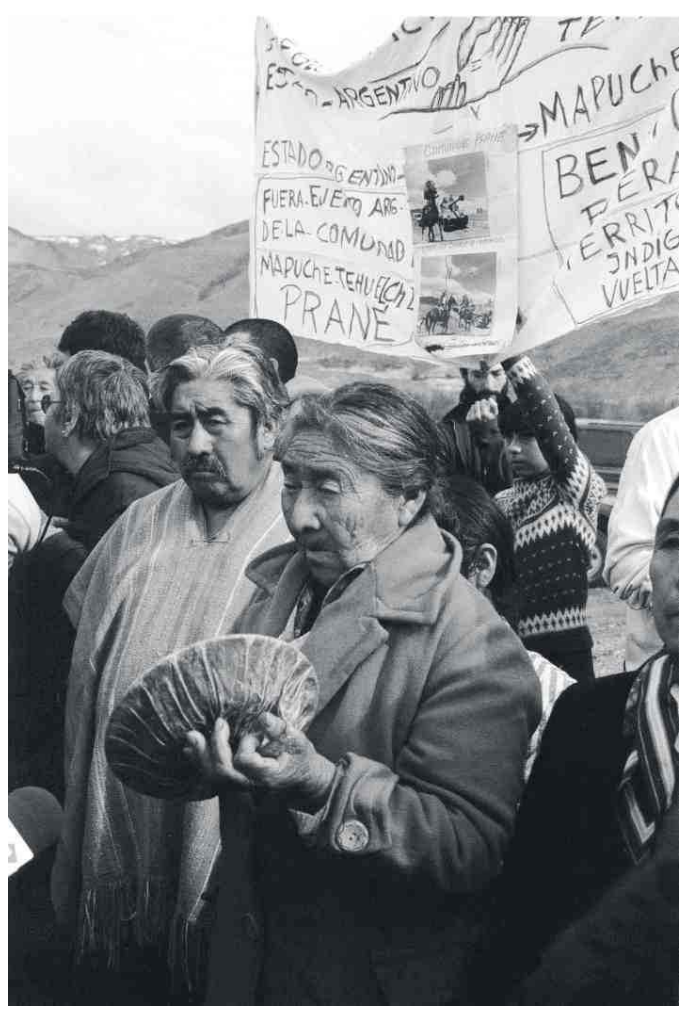

(c) Renata Biernat 
1 En tant qu'historien j'ai participé, entre 1999 et 2000, aux côtés d'ethnologues, d'archéologues et de muséographes - membres d'universités argentines ou d'organismes de recherche - à l'équipe responsable des collections et du scénario historique du musée Leleque, situé au pied de la cordillère des Andes, dans la province du Chubut, proche de la frontière avec le chili.

2 Il s'agit d'une expérience inédite en Argentine d'un musée ouvert au public et géré par un groupe privé. Construit au milieu d'une estancia d'élevage qui porte le même nom, le musée Leleque a été financé par le groupe Benetton, propriétaire de vastes étendues de terres en Patagonie argentine. La collaboration des scientifiques avait pour but de restituer aux populations locales les connaissances produites par les différents projets que ceux-ci avaient réalisés, parfois plusieurs années avant de souscrire à l'accord de collaboration avec le groupe Benetton en octobre 1997. En contrepartie, le mécène consacra une petite partie du budget du musée à la création d'un centre de recherche. Ainsi avons-nous reçu, chercheurs et étudiants, une aide financière de la part du groupe industriel, lequel a également financé la réalisation de campagnes archéologiques, le travail d'archives, la participation à des congrès, l'acquisition d'ouvrages et l'édition de publications ${ }^{1}$. Mais le musée est aussi le fruit de travaux antérieurs sur la Patagonie septentrionale réalisés par les différents chercheurs du groupe grâce au financement de l'État argentin qui, depuis les années 1980, a soutenu différents programmes et versé de nombreux salaires.

3 L'exposition inaugurale s'intitula Patagonia 13000 años de historia. Ce titre, qui renvoie à la plus ancienne datation archéologique connue, faisait de celle-ci le point de départ du récit du passé humain de la région. À l'intérieur de cette longue chronologie, deux thèmes se partagèrent le devant de la scène: d'une part, les conditions de la spoliation et de l'ethnocide des communautés indigènes depuis l'occupation du territoire par la nation argentine à la fin $\mathrm{du} \mathrm{xIX}^{\mathrm{e}}$ siècle et, d'autre part, les modalités de l'implantation économique et sociale des populations d'immigrants chiliens, européens et libanais au lendemain de l'expansion nationale vers le sud.

4 Une partie importante de cette exposition s'appuyait sur des photographies ethnographiques anciennes, sur des clichés pris par des ingénieurs topographes en campagne avec l'armée argentine dans le nord de la Patagonie en 1882 et 1883, sur des portraits d'indigènes prisonniers à Buenos Aires au cours des années 1880 et sur des photographies faites par le missionnaire italien de la congrégation salésienne Alberto De Agostini dans l'extrême sud du continent américain, entre 1910 et $1930^{2}$.

5 Nous proposons ici une réflexion critique sur l'utilisation muséographique et historiographique de ces images au cours de cette expérience, ainsi que sur les débats et les différentes interprétations que leur circulation suscita, plus particulièrement sur les tensions à l'œuvre dans un musée qui, financé par de grands propriétaires fonciers étrangers, veut faire le récit de l'expropriation et du remaniement du contrôle indigène sur le territoire.

6 Attentif aux conflits judiciaires sur la propriété de terrains proches du musée qui éclatèrent au lendemain de son inauguration entre le groupe Benetton et plusieurs familles mapuche, cet article s'interroge sur la dimension éthique, les limites et les possibilités pour les chercheurs en sciences sociales et les institutions publiques ou gouvernementales de participer à des initiatives privées qui produisent les récits et les nouveaux imaginaires de l'identité ethnique. Comme en Amérique du Nord, «[...] qui a 
[elle aussi] une expérience colonisatrice intérieure, [...] la proximité des uns et des autres tend à faire du musée une arène symbolique de luttes de pouvoir et d'identités ${ }^{3}$ ». À la manière d'un film qui raconterait le tournage d'un autre film, il est intéressant de mettre en regard les photographies de la fin du XIX ${ }^{e}$ siècle exposées au musée Leleque avec celles de l'inauguration du musée lui-même et de voir, dans ce jeu de miroirs, se dessiner les différents niveaux de représentation et émerger le méta-discours des pratiques muséographiques.

\section{Le projet initial du musée Leleque}

7 Sous la direction de María Teresa Boschín, archéologue spécialiste du nord de la Patagonie, la première exposition entendait rendre compte de 13000 ans d'histoire, organisés en petits fragments narratifs. Le prospectus destiné aux visiteurs la résumait ainsi : « Le musée Leleque raconte l'expérience des peuples indigènes et immigrants de la Patagonie, depuis la chasse aux guanacos ${ }^{4}$ avec des projectiles en pierre jusqu'à l'élevage lainier dans les champs clôturés. » Avec une prétention certaine de totalité puisque la période couverte va du paléolithique à l'époque moderne, le récit combinait diachronie et interprétation anthropologique pour montrer, de manière didactique, les changements opérés par les sociétés qui ont peuplé le territoire dans ce large cadre régional et temporel.

8 L'exposition se divisait en quatre salles. La première, intitulée " les peuples originaires ", offrait une analyse des objets archéologiques antérieurs à l'arrivée des Européens au XVI ${ }^{\mathrm{e}}$ siècle et présentait les particularités du mode de vie des chasseurs-cueilleurs ainsi que leurs représentations idéologiques cristallisées dans l'art rupestre. La salle II, «le contact ", rendait compte de l'impact dans les consciences indigènes et européennes de l'arrivée des Européens dans l'extrême sud du continent. Elle traitait également des relations inter-ethniques, caractérisées aussi bien par des échanges matériels et culturels actifs que par des affrontements qui ne prirent fin qu'avec l'intervention armée des États argentin et chilien pendant le dernier quart du XIX ${ }^{e}$ siècle. Les salles III et IV, intitulées respectivement «immigration, terre et capitaux» et "la société patagonique», prolongeaient le récit jusqu'aux années 1930 en offrant une interprétation du processus postérieur à la défaite des indigènes, de leur marginalisation sociale croissante, de la présence de l'État, de l'incorporation finale de la Patagonie dans le système capitaliste et, enfin, du phénomène de l'immigration. Cette interprétation était fondée sur les conflits pour le contrôle de la terre et des ressources économiques qu'elle générait.

Du point de vue méthodologique, nous avions d'abord élaboré le scénario en définissant l'histoire que nous voulions raconter puis, dans un second temps, nous avions organisé les collections et leur exposition en fonction des axes du récit retenu. De manière générale, la participation des habitants de la région fut considérable. Ils mirent à notre disposition leurs histoires familiales en nous prêtant documents, photographies et objets qui ont fini par composer une grande partie des collections. 


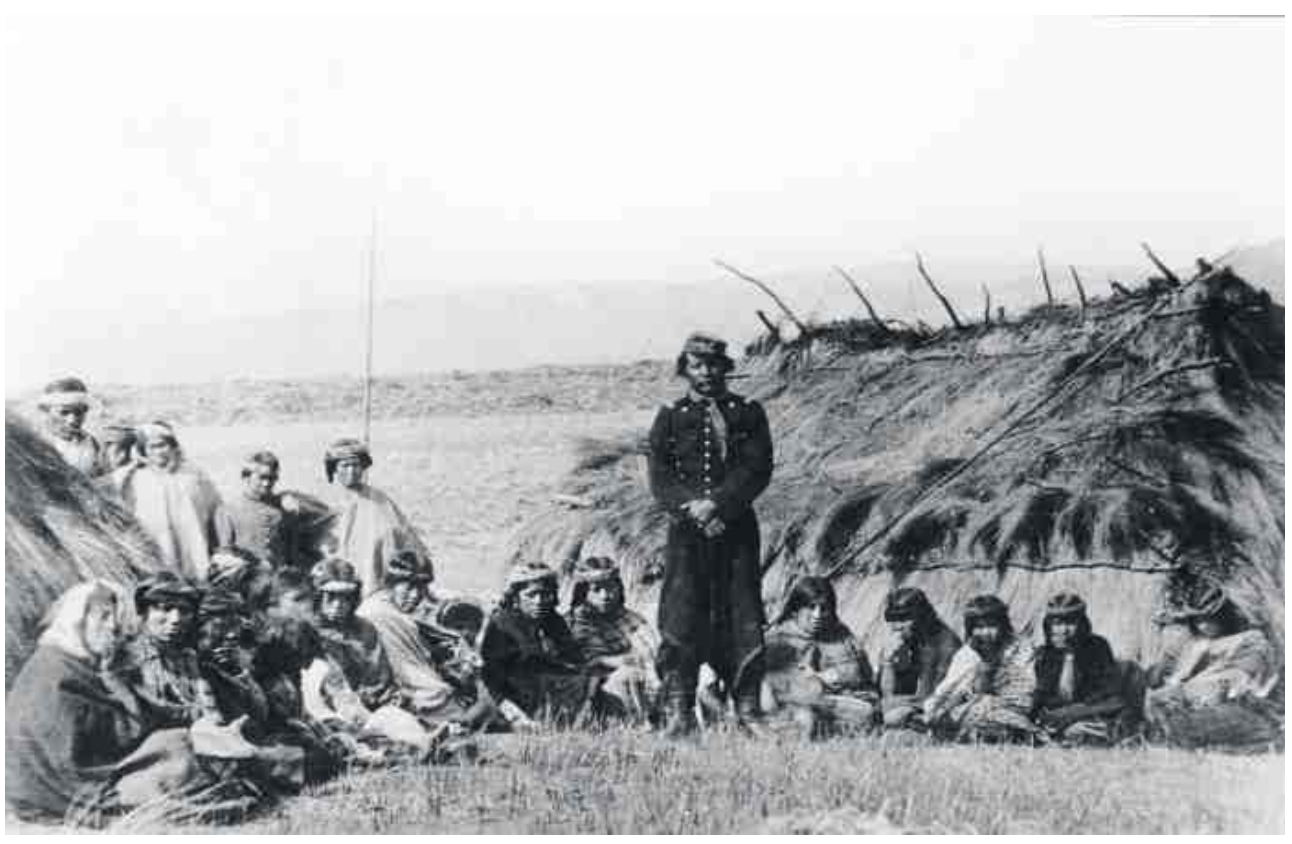

(C) Encina, Moreno y Cía l'équipe scientifique ont toujours été récoltées avec l'accord des personnes concernées, on ne peut pas en dire autant de celles spécifiquement collectées pour le musée Leleque. Si l'enthousiasme et l'implication des habitants descendants d'Européens et de Libanais furent réels, cela n'a pas été le cas de la participation indigène. Dans le cadre d'une représentativité segmentale caractéristique de ces organisations, certains membres des communautés indigènes ont expressément soutenu la réalisation du musée Leleque, soit au nom des relations personnelles préexistantes avec les scientifiques, soit en fonction de diverses stratégies politiques et familiales quant aux avantages et aux inconvénients qui pouvaient surgir de la collaboration culturelle à un projet dirigé par Benetton. Mais bien qu'il n'y eût pas de refus manifeste avant l'inauguration, le désintérêt des indigènes envers un musée qui entendait raconter leur histoire, solidaire de leur cause mais sans pour autant les consulter sur la pertinence de l'initiative, l'emportait clairement. La convocation des communautés n'a pas été assez large. Rosa Chiquichano - avocate tehuelche actuellement députée péroniste de la province du Chubut - a été la seule indigène à participer à la commission honoraire du musée.

11 Sans doute qu'au-delà des désaccords et des différends qui ne manqueraient pas de surgir au sein de l'équipe, l'intérêt pour mener à bien le projet semble l'avoir emporté parmi les scientifiques. Ceux-ci n'en demeuraient pas moins conscients que la pleine participation des communautés indigènes à une initiative venue de grands propriétaires terriens serait une contradiction insurmontable qui générerait de tels conflits éthiques et politiques que l'équipe du musée devrait abandonner ses objectifs de départ pour ne pas échouer.

Il faut également mentionner la collaboration de différentes institutions publiques entre autres celle du musée ethnographique de l'université de Buenos Aires - et l'aide fournie par plusieurs organismes nationaux et provinciaux. Suite à l'accord avec Benetton, les premières actions pour mettre en place le futur musée débutèrent en 1997. 
Jusqu'à la date de l'inauguration, le 12 mai 2000, les travaux des scientifiques et ceux du centre de recherche annexe se sont développés sans la moindre incitation idéologique. Pendant cette période, le mécène a fait preuve d'une ingérence minime dans les aspects formels et visuels de l'exposition, ainsi que dans la réalisation graphique des prospectus. En revanche, tout son intérêt s'est concentré sur l'organisation de l'inauguration ellemême, fidèle aux préoccupations d'une grande entreprise transnationale dont la principale manière d'être présente sur le marché consiste justement à y vendre une image. Pour le dire vite, la forme devait venir supplanter le contenu. L'argument publicitaire incita les designers de Benetton à remplacer la mosaïque de photographies des colons venus de tous horizons que nous avions sélectionnées par le portrait solitaire de Kopacho, un cacique du sud des années 1920. Bien enveloppé de son quillango en cuir de guanaco, les cheveux attachés, Kopacho se révélait être l'icône synthétisant et signifiant le désert et la Patagonie.

Localisation du musée Leleque en Argentine.

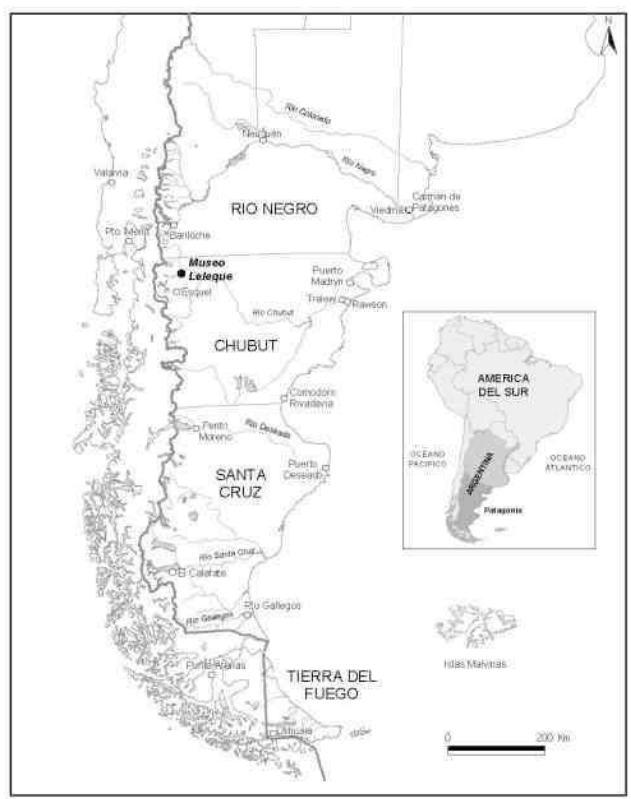

Dans ce climat de relatif désintérêt de l'entrepreneur pour le contenu que nous élaborions, nous n'avons subi ni la moindre contrainte ni le moindre contrôle de notre propos. Cependant, la nécessité pour les scientifiques de maintenir un équilibre difficile entre le caractère idéologique de la proposition et le contexte pratique dans lequel celleci se formait contribua à l'émergence d'une exigence implicite quant aux limites chronologiques à fixer. Michel de Certeau (1978) a démontré que l'«opération historiographique " s'articule rétrospectivement, du présent au passé, bien que la narration l'inverse et se présente dans un ordre contraire. La séquence de l'histoire argentine ayant été arrêtée dix années avant l'émergence du péronisme et au moment des grands affrontements de classes pendant les années 1940, les frères Benetton et leur entreprise demeuraient à l'extérieur de la problématique tracée par le musée, si ce n'est par une série d'allusions indirectes aux effets sociaux de la grande propriété foncière. 
Acquise par le groupe au début des années 1990, l'Argentine Southern Land Co. était financée à l'origine par des capitaux appartenant à des personnes proches de la couronne d'Angleterre, qui bénéficièrent, à la fin du XIX ${ }^{\mathrm{e}}$ siècle, de l'attribution de grandes étendues de terres aux dépens des familles indigènes alors en passe de perdre le contrôle de la Patagonie. Observateurs soi-disant impartiaux du passé, les nouveaux propriétaires italiens du Xxi ${ }^{e}$ siècle ne pouvaient que s'émouvoir des grandes tragédies locales d'antan telle la répression des ouvriers ruraux pendant les grèves des années 1920-1922 -, elles aussi racontées dans le musée.

14 La proposition originale quant au rôle éducatif du musée - à l'adresse principalement des écoles rurales où sont scolarisés des enfants indigènes - ainsi que son programme de diffusion culturelle conçu comme un cadre de confrontation et de multiplication des débats politiques et sociaux n'ont fait qu'amplifier les ambiguïtés et les contradictions entre le récit historique, les pratiques muséographiques et le lieu institutionnel où ils étaient produits. Peu à peu, notre comportement en venait à friser la schizophrénie autoréférentielle. En d'autres termes, un musée voué à la narration du conflit social, à un moment ou à un autre, finirait par le générer. D'une certaine manière, ce travail semblait un discours sur nous-mêmes, sur cette équipe de scientifiques et de muséologues dans sa relation avec le mécène d'une part, avec les sujets historiques que nous objectivions comme référents d'autre part.

Il fallut peu de temps après l'inauguration du musée Leleque pour que cette focalisation sur le conflit social devienne insupportable à l'imaginaire « naïf » de la Patagonie, perçue comme terre promise, terre de rêves et de liberté, représentation à la mode que l'on trouve dans les médias et les agences de tourisme, mais aussi dans le conformisme d'une partie non négligeable de la population locale. Cet imaginaire à large diffusion, qui colle exactement au multiculturalisme publicitaire des United Colors of Benetton, fait de l'indigène l'élément authentique de la Patagonie en l'articulant harmonieusement avec la nature, en le vidant de ses tensions et en l'intégrant dans cet ensemble idyllique d'identités et de classes articulées à l'intérieur de l'espace national. Très vite, il fallut trancher entre un musée qui fait écho aux problématiques sociales et un musée qui met les indigènes en vitrine, édulcore la mémoire collective et légitime les politiques d'entreprise. 
Baptême des Indiens de Reuquecura en Codihué, 1883.

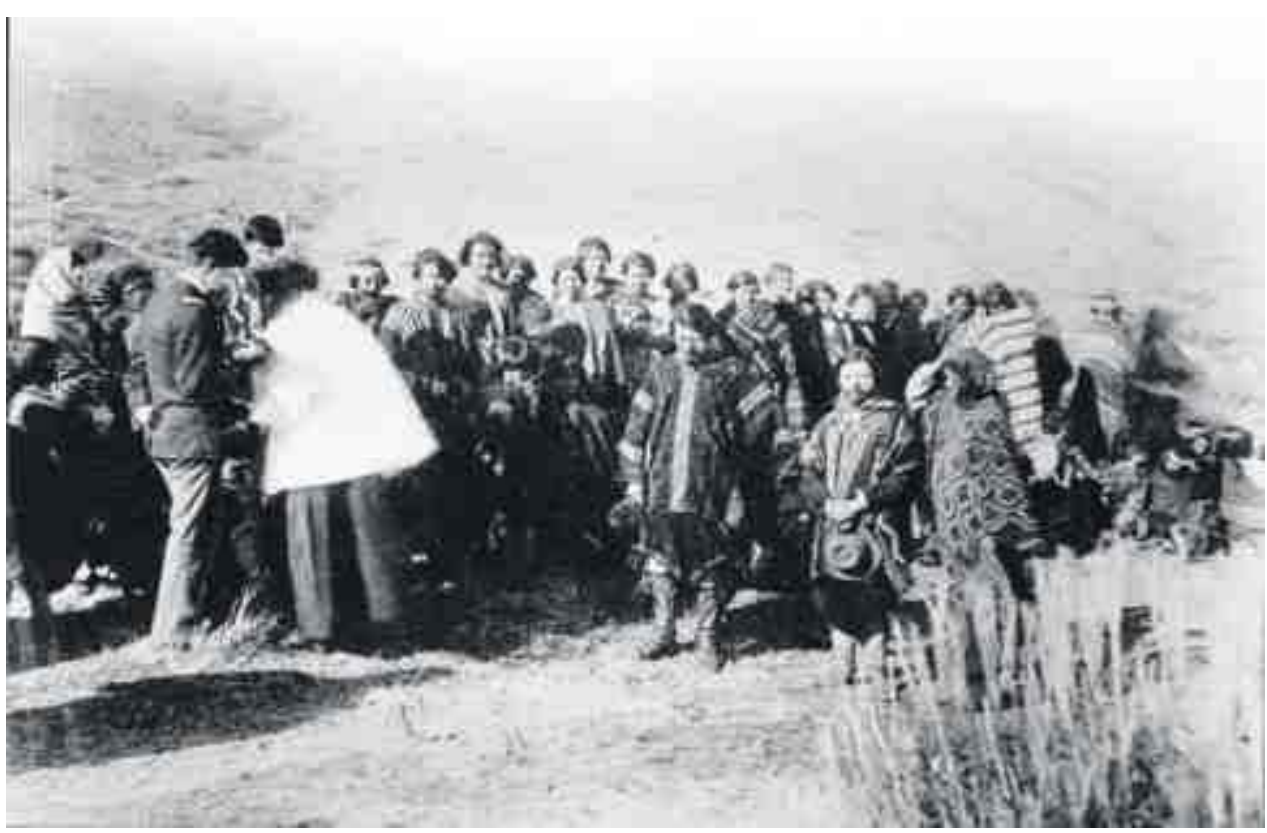

(c) Encina, Moreno y Cía

Baptême du musée Leleque, 12 mai 2000.

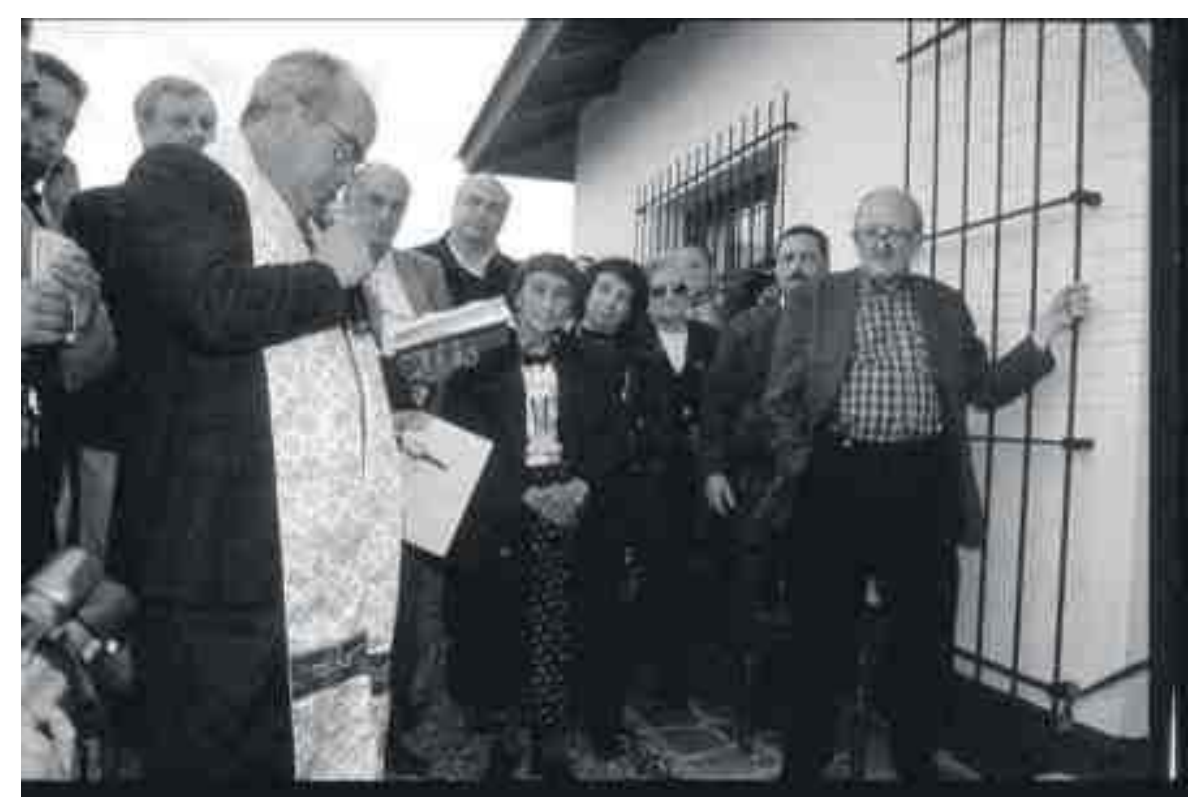

(C) Luis Abregú

Ainsi, l'exposition d'une photographie d'un cacique indigène et de sa famille, prise immédiatement après qu'ils se soient rendus aux troupes argentines en décembre 1882, irait à l'encontre de la volonté de l'équipe scientifique, pourtant bien décidée à montrer la catastrophe. Malgré la légende de la photographie qui insistait (sans que cela soit nécessaire) sur son caractère dramatique, le phénomène Benetton semblait changer la signification de n'importe quel énoncé, fixant le conflit inter-ethnique dans un coin perdu du passé, écartant les relations de pouvoir et de domination et exonérant les 
propriétaires blancs et les autorités nationales de leurs responsabilités actuelles. Mais l'aura benjaminienne eut le dernier $\operatorname{mot}^{5}$. Le portrait de la famille du cacique Millamain révéla rapidement une capacité persistante à produire du sens, en montrant que malgré la légitimité juridique des titres que les propriétaires des champs s'empressaient de faire valoir, l'occupation des territoires de la Patagonie s'était fondée sur les événements violents $\mathrm{du} \mathrm{xIX}^{\mathrm{e}}$ siècle et sur l'exclusion sociale consolidée au $\mathrm{xx}^{\mathrm{e}}$ siècle. Comme le préconise Jean-Loup Amselle à propos du cas africain (Amselle et Dupuis 1999: 477), la relation entre «musées » et "violence » doit aussi se montrer dans toute son ampleur aux confins de l'Amérique du Sud ${ }^{6}$.

\section{Les points de vue indigènes}

17 Les tensions sous-jacentes à la création d'un musée retraçant l'appropriation des terres indigènes et financé par une entreprise à capital européen, qui plus est très médiatique et surtout propriétaire de grandes estancias d'élevages, ne se donnèrent à voir que lors de la cérémonie d'inauguration, le 12 mai 2000. Sans avoir été invités, une vingtaine d'indigènes issus de familles $d u$ nord-est $d u$ Chubut et organisés socialement et politiquement en communauté profitèrent de l'occasion pour se faire entendre des autorités nationales et provinciales présentes lors de la cérémonie. Ils n'auraient pu trouver meilleure occasion pour faire connaître leurs revendications à la centaine de journalistes argentins et étrangers conviés par les organisateurs, transportés et logés à Leleque et à Esquel, à plus de 1800 kilomètres de Buenos Aires, et ce aux frais de ces derniers.

Comme nous l'avons indiqué, les experts en communication et en relations publiques du groupe Benetton concentrèrent tous leurs efforts sur la préparation de la cérémonie inaugurale. S'y pressaient des gens de télévision nord-américains et européens, de grandes figures académiques argentines, les directeurs de divers musées, des membres du Secretaría de Cultura directement dépendant de la Presidencia de la Nación, des officiels de l'armée et de la gendarmerie nationale, le gouverneur de la province du Chubut, tous mêlés aux habitants de la Patagonie qui avaient participé à la mise en musée de leur histoire de vie.

19 Pendant que le prêtre du Maitén, village voisin de quelque 2500 habitants qui se trouve au cœur d'une autre estancia des frères Benetton, donnait sa bénédiction à l'ouvrage, les manifestants, à environ soixante mètres de là, lancèrent des cris de protestation en langue mapudungun pour exprimer leur rejet de l'événement. Les indigènes brandissaient des pancartes qui accusaient Benetton et l'État argentin d'être « complices dans l'usurpation du territoire mapuche ». Leurs vêtements contrastaient avec les uniformes de gala des soldats du $3^{\mathrm{e}}$ Régiment de cavalerie, qui se régalaient alors d'un lunch offert pour agrémenter l'inauguration. Cent vingt ans plus tôt, cette même division militaire avait joué le premier rôle dans la guerre contre les indigènes du nord de la Patagonie. Malaise et confrontation pesèrent largement sur l'ambiance générale, mais ne mirent pas pour autant fin à la cérémonie inaugurale. Au contraire, celle-ci se poursuivit, sans se soucier des protestations.

20 La scène aux allures postmodernes, pour se faire plus complète encore, comptait parmi les invités Josefina Braun, femme de confiance du personnel de Benetton en Amérique latine, issue d'une famille qui a accumulé depuis 1890 une fortune colossale en Patagonie 
grâce au commerce et à l'élevage. Josefina, vêtue d'un poncho mapuche, essaya de persuader les manifestants de l'importance du musée Leleque pour la diffusion du passé indigène. Pendant l'échange coloré de répliques, elle avança que le groupe Benetton avait acheté la terre en toute légalité, que les conflits pour sa possession dataient de plus longue date, qu'ils n'avaient rien à voir avec le groupe. D'un autre côté, elle promit d'en référer à ses employeurs pour qu'ils prennent en considération les réclamations.

Manifestation indigène lors de l'inauguration du musée Leleque, 12 mai 2000.



(c) Luis Abregú

21 Comme le raconte Moira Millán, werken ou "messagère " de la communauté Pillán Mahuiza, le groupe d'indigènes parvint à se frayer un chemin jusqu'à l'estanciaoù s'élève le musée «[...] malgré l'important dispositif de sécurité, en disant qu'ils venaient le soutenir. Il y avait des journalistes du monde entier. Ils nous disaient: "C'est fantastique !" Chaque fois qu'ils allaient couper le ruban, nous jouions de nos instruments en disant: "Que les Benetton nous rendent la terre !" "

Leur apparition sur la propriété - traversée en fait par une voie publique pour laquelle Benetton doit servitude de passage - concentra toute l'attention des journalistes, qui abandonnèrent la conférence de presse où se succédaient les discours des membres de l'équipe scientifique, du représentant légal de la Compañía de Tierras Sud Argentino, de la directrice nationale du Patrimonio Histórico y Museos, de la cacica Lucerinta Cañumil venue spécialement de la province voisine du Río Negro pour octroyer le consensus indigène au musée - et du gouverneur d'alors de la province du Chubut, José Lizurume. Après une première tentative infructueuse, l'élu provincial parvint à dialoguer avec les manifestants qui exprimaient le message suivant: « Nous ne sommes pas des objets de musée : nous sommes vivants et tous les jours un peu plus pauvres. Nous voulons être écoutés. Le gouverneur vient assister à ce cirque mais nous, il ne veut pas nous recevoir ${ }^{8}$ . » 
Cacica Lucerinta Cañumil, 2000.

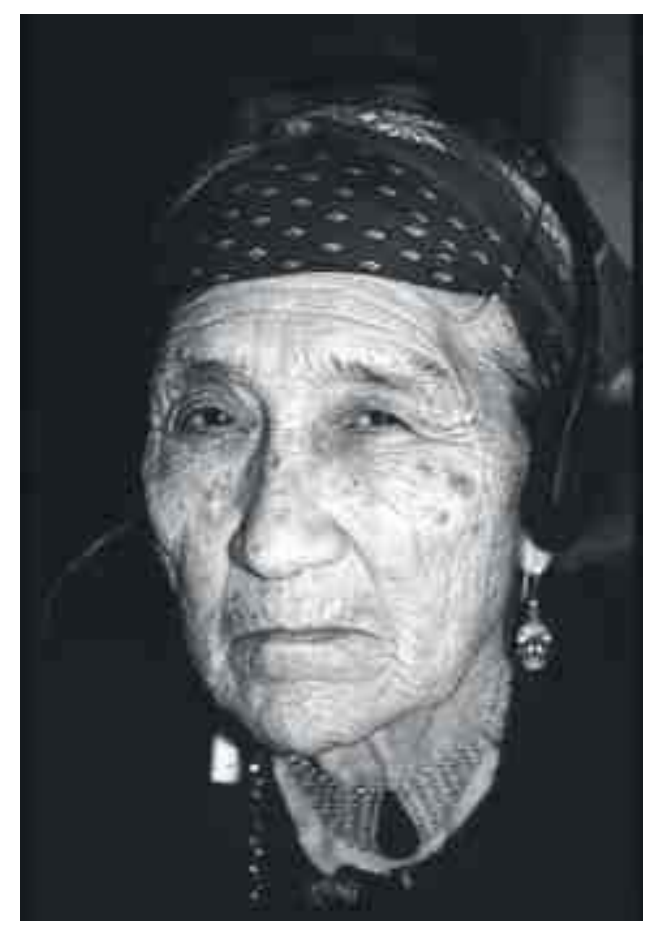

(C) Luis Abregú

De même, Luis Millán, évoquant l'enjeu de la manifestation pour les indigènes, expliqua que, pour eux, «l'inauguration ne signifiait rien du tout ${ }^{9} »$. Sa sœur Moira précisa devant la presse: "Nous n'attaquons pas le musée. Parce que nous croyons à la diversité culturelle. Nous allons nous retirer, nous ne voulons pas d'assistanat, nous réclamons justice $^{10}$. $\gg$ La majorité des manifestants n'accepta pas l'invitation quelque peu tardive qui leur fut faite de visiter les salles du musée et l'exposition. Comme il ressort des propos de Moira Millán, le rejet par les indigènes de l'initiative culturelle lancée ce jour-là ne fut pas frontal, peut-être en raison du constat tactique que le musée semblait emporter l'adhésion d'une grande partie de la population locale. Conscients du jeu de représentation institué par toute pratique muséographique, ils recommandèrent à leurs interlocuteurs qui voudraient «[...] voir la culture des natifs de ne pas aller au musée, [mais d'aller voir leurs] communautés ${ }^{11}$ ». Dans tous les cas, le musée Leleque ne revêtait pas une très grande importance à leurs yeux. Il est clair que le cœur de la manifestation et du conflit résidait ailleurs.

Entre deux salles du musée se trouvait un écriteau citant une phrase de la chronique de Guillermo Cox, un aventurier chilien qui traversa la cordillère des Andes pour rejoindre la Patagonie en 1863. La phrase de Cox (1863: 108) semblait parler de cette journée d'inauguration et de la situation générale du musée : «[...] si les Indiens ont été conviés par les Espagnols au banquet de la civilisation, ils n'eurent que peu de place à table. »

De même que dans n'importe quel débat politique et culturel, les communautés indigènes ne présentèrent pas un front homogène. Prétendre à une réaction unique consisterait à les substantialiser, quand leurs modes et leurs logiques de représentation sont divers et complexes. Il convient de parler de points de vue indigènes au pluriel. Leurs interprétations, leurs obligations et leurs attentes différaient. J'ai déjà fait mention de Rosa Chiquichano, ainsi que de la cacica Lucerinta Cañumil, laquelle était 
particulièrement préoccupée par la suspension du financement par l'État de l'enseignement en langue mapudungun. Les femmes d'un atelier artisanal appelé RucaLamngen, «maison des sœurs » de Colonia Cushamen, village indigène de 700 habitants encerclé par les propriétés de Benetton, agirent dans le même sens. Par ailleurs, le musée présentait pour les artisans une occasion rare de vendre leur production textile aux touristes, à travers le seul programme social qui vit le jour et qui existe encore aujourd'hui à Leleque. Certains ouvriers agricoles et autres employés indigènes de l'estanciaassistèrent à la cérémonie organisée par leur patron.

Ouvrier agricole de l'estancia Leleque et sa famille, 2000.

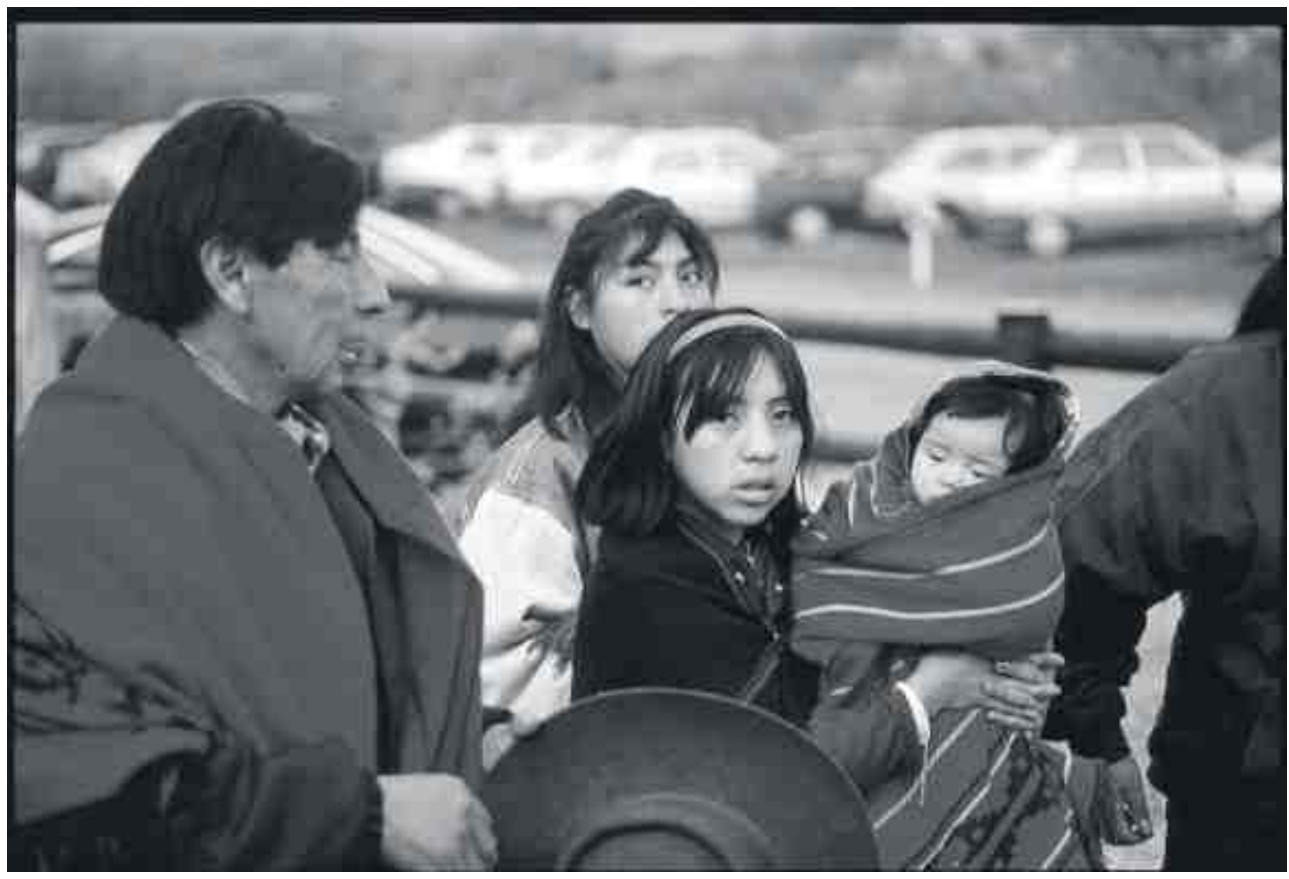

(C) Luis Abregú

Lors de l'inauguration, différentes protestations convergèrent. Le groupe «11 de Octubre ", dont le nom fait référence au «dernier jour de liberté », à savoir la veille du débarquement de Christophe Colomb aux Antilles, répudia Benetton et proclama la vigueur de la « Nation mapuche-tehuelche » face à l'État argentin. Dans le même ordre d'idée, José Prane dénonça l'occupation par l'armée argentine de la "Legua 4 », une fraction de la réserve indigène de Nahuelpan, à 80 kilomètres de Leleque. D'autres familles, accompagnées par leur avocat, rendirent publics les litiges juridiques en cours sur l'expropriation de terres (bien moins étendues que celles acquises par les Benetton) opérée par des descendants d'immigrants libanais dans les environs de Vuelta del Río.

Il n'y eut pas non plus unanimité à propos des choix muséographiques, pas plus que sur la publication des photographies des indigènes prises au lendemain des défaites du XIX siècle. Certains membres des communautés s'en réjouirent, d'autres les remirent en question. Lors de la présentation d'un livre qui reproduit ces photos (Vezub 2002), Joaquín Antieco, sous-officier de la police de la province du Chubut et fils de l'autoproclamé «créateur du drapeau du peuple mapuche-tehuelche » que portaient les manifestants, rejeta ces images jusqu'alors inédites de la profanation de chenques, ou cimetières indigènes. Bien que plusieurs travaux à la louange des guerres contre les 
Indiens, présentées comme l'avancée de la "civilisation» sur la «barbarie », soient illustrés par les photographies des ingénieurs topographes qui accompagnèrent les troupes militaires en 1882 et 1883 , toutes les publications précédentes prirent le soin d'éviter les images des tombes excavées par des indigènes sur ordre des soldats ${ }^{12}$. Ces images, en particulier celle d'un cacique «collaborationniste » vêtu de l'uniforme argentin et qui mobilise ses propres parents pour procéder à la profanation, semblent avoir définitivement écarté tout recours à un quelconque essentialisme. Les différents malaises des indigènes actuels - les uns rejetant l'État, les autres les fonctionnaires de police - expriment la complexité et la diversité des prises de position face au musée et aux photographies.

Cimitière indigène ou chenque de la tribu de Reuquecurà, 1883.

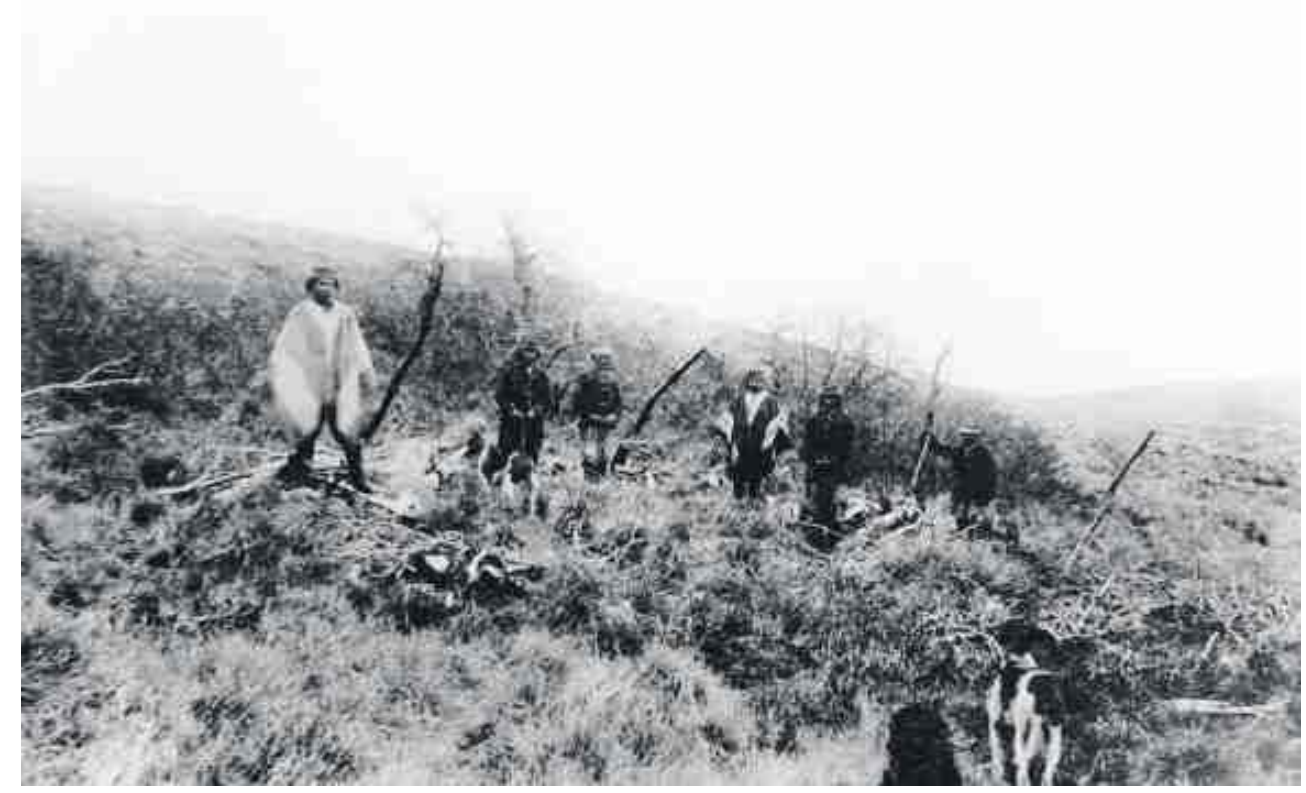

(c) Encina, Moreno y Cía 


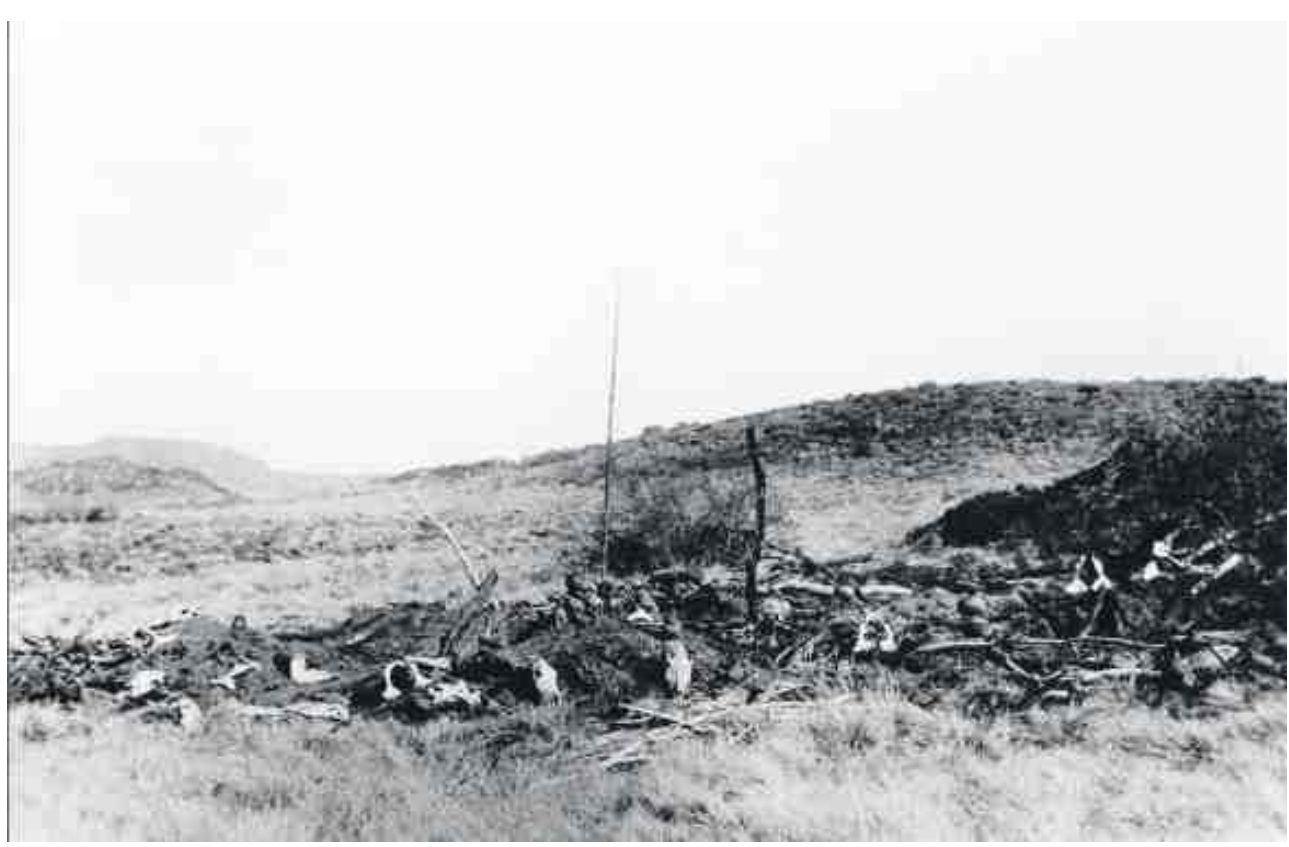

(c) Encina, Moreno y Cía

\section{Réclamation de la terre, identités ethniques et légitimation patronale}

Les événements du jour de l'inauguration marquèrent une rupture. Depuis, le groupe Benetton a recadré son projet muséographique. Le conflit entre les intérêts de l'entreprise, les préoccupations des scientifiques et celles des habitants qui avaient accompagné le projet est devenu plus explicite. Les objectifs de départ ont été modifiés, la mission sociale et culturelle du musée déplacée, les projets du centre de recherche restreints. Les réponses des scientifiques engagés dans sa mise en place se sont avérées antagoniques. La logique patronale a gagné les interstices des intérêts divergents des différents chercheurs pour finir par imposer ses propres critères.

Les premiers désaccords portèrent sur l'ampleur du programme éducatif et les moyens dévolus aux visites scolaires, ainsi que sur la suppression du programme social, exclusivement limité depuis lors à la vente de l'artisanat indigène. Du point de vue de l'entrepreneur, le musée est devenu une vitrine pour le tourisme et la presse, destiné à contrebalancer la mauvaise publicité faite par les nombreux conflits avec les communautés et les instances gouvernementales, provinciales ou municipales.

Le discours historiographique sur un passé complexe et la proposition muséographique de multiplier les débats et les interlocuteurs n'ont pu dépasser leurs propres ambiguïtés. L'idée d'un musée ouvert et participatif, capable de contourner les limites et les contradictions que le projet initial portait en son sein, fut abandonnée. María Teresa Boschín a été écartée de la direction du musée Leleque à la fin de l'année 2002 et l'ethnologue Rodolfo Casamiquela, président de la Fundación Ameghino, a pris en charge la gestion quotidienne et les choix plus globaux du musée. Pour protester contre ce 
changement de cap, de nombreuses familles des provinces du Río Negro et du Chubut qui avaient prêté objets, photographies et souvenirs ont exigé qu'ils leur soient rendus.

Les affrontements pour la propriété de la terre ont continué, parallèlement aux péripéties du musée. Ce conflit a d'ailleurs pris une tout autre ampleur après 2002 quand la Compañía de Tierras Sud Argentino, propriété des Benetton, engagea une poursuite judiciaire pour usurpation contre les frères Atilio et Rosa Curiñanco afin de les déloger du domaine de «Santa Rosa» qu'ils occupaient depuis quelque temps ${ }^{13}$. En mai 2004, les frères Curiñanco ont été définitivement acquittés des charges de délit et de responsabilité pénale retenues contre eux, mais les indigènes durent quitter les 535 hectares du domaine. Le juge correctionnel Jorge Eyo finit par repousser les arguments de la défense, qui soutenait que la parcelle en question était une terre fiscale, et, de ce fait, susceptible d'être occupée et valorisée par la famille mapuche.

Après un tel échec judiciaire, des négociations eurent lieu entre les différentes parties, avec pour médiateur le prix Nobel argentin de la paix Adolfo Pérez Esquivel, reconnu pour son travail de défense des droits de l'homme pendant la dictature militaire. Les frères Benetton et les frères Curiñanco s'entretinrent à Rome, ces derniers étant accompagnés de leur avocat et de Mauro Millán, du groupe " 11 de Octubre ». Si les entrepreneurs offrirent quelque 2500 hectares de terre exploitable dans les environs d'Esquel, au pied de la Cordillère, les négociations furent infructueuses en ce qu'elles ne purent définir le statut de cette remise de terre. Le groupe entrepreneur offrait une «donation» ou une «cession» avec Pérez Esquivel comme dépositaire ou garant. Les indigènes exigeaient eux la "restitution " ou la "dévolution ", tout en étant disposés à accepter du gouvernement argentin qu'il soit le responsable du transfert. La différence était significative, surtout comme précédent pour de futurs litiges. À l'occasion de la grande campagne de presse qui accompagna les négociations à Rome, Luciano Benetton déclara que la proposition constituait une «[...] contribution concrète tout autant que symbolique vers une solution aux luttes historiques des populations indigènes ${ }^{14} »$. Selon les arguments de l'entrepreneur italien, largement fondés sur le nouveau discours historique du musée, le problème social se réduisait à une séquelle du passé, à un problème qui ne le concernait pas puisqu'il se posait selon lui entre les indigènes et non avec les indigènes. 


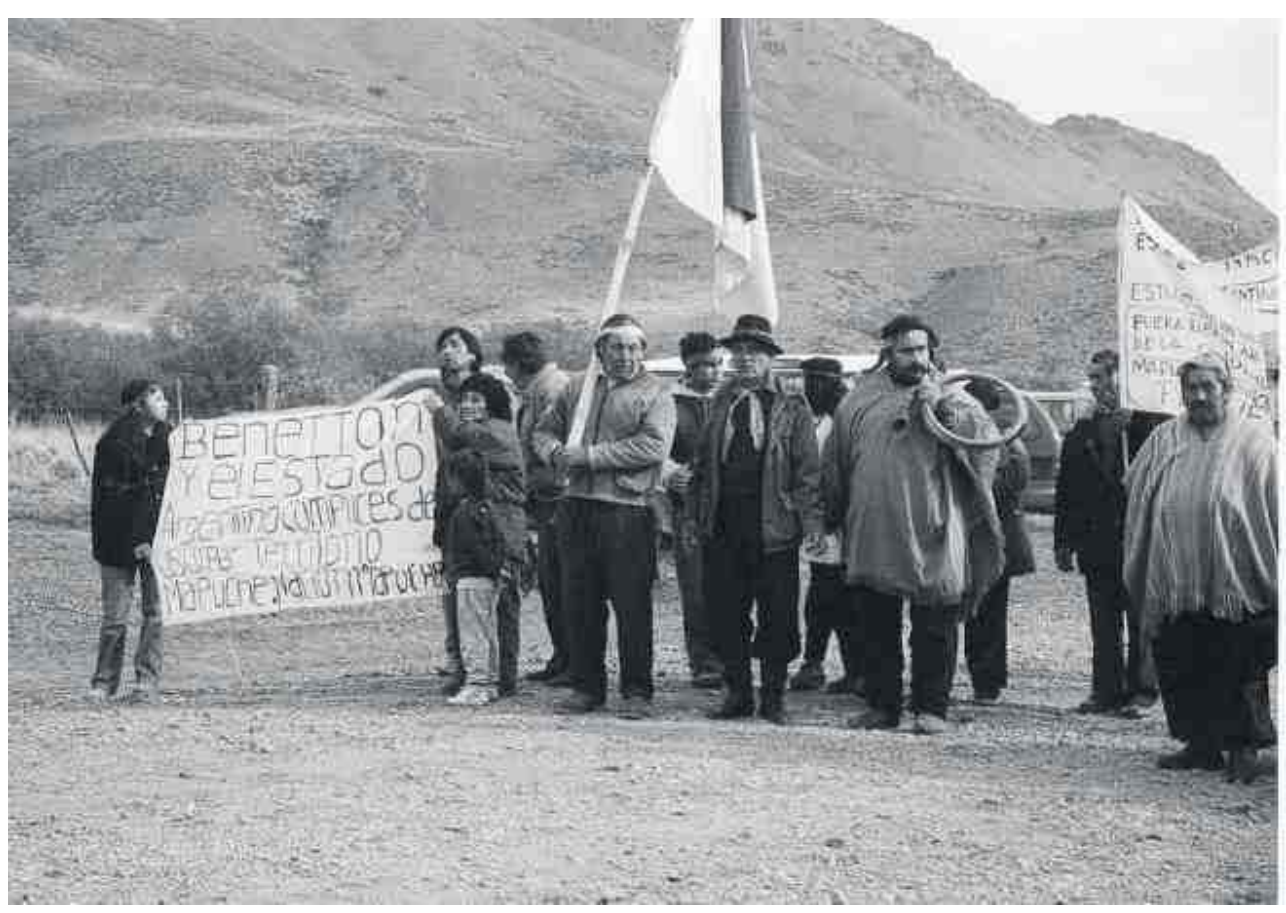

(C) Renata Biernat

Après une fermeture de presque un an en raison du problème de la restitution des collections réclamées par les familles et de la volonté de modifier entièrement le projet initial, le musée Leleque rouvrit ses portes en mars 2004. Les présupposés ethnocentriques de la nouvelle version s'appuient sur les recherches d'ethnologie traditionaliste de Rodolfo Casamiquela (1965, 1969, 1985 et 1995). Si les principaux points de la pensée essentialiste de l'auteur étaient déjà visibles dans ses publications des dernières décennies, leur transposition dans le discours muséographique a permis de les vider de toute nuance sur les phénomènes de métissage et d'hybridation culturelle et de leur donner par là une dimension nettement instrumentale, tout à fait compatible avec les intérêts des entrepreneurs.

L'opération discursive simplifie désormais considérablement le processus historique de construction des identités en Patagonie. En cohérence avec la pensée de Casamiquela, les Tehuelche sont originaires d'Argentine et les «Araucanos » ou Mapuche viennent du Chili. Il existe pourtant un consensus parmi les anthropologues et les historiens actuels pour affirmer que la cordillère des Andes était socialement poreuse et que, jusqu'aux dernières décennies qui précédèrent l'expansion des États-nations, les frontières juridiques étaient dénuées de toute signification pour des populations indigènes étroitement liées par la parenté et le commerce de part et d'autre de la chaîne montagneuse. Cette dernière perspective, en soulignant les effets d'interaction et de contact, et en mettant en cause la naturalisation d'une limite raciale ou culturelle sur la cordillère, ne néglige pas l'importance des conflits ou l'émergence d'identités régionales lesquelles, dans tous les cas, se configurèrent sur la base de processus sociaux et politiques complexes et non à partir du suivi d'une liste fermée d'attributions ethniques préétablies ${ }^{15}$. 
35 À l'ethnologie essentialiste s'ajoute une pointe de xénophobie nationaliste. Casamiquela soutient que les Mapuche sont d'origine étrangère, «chilienne », et qu'ils ne seraient arrivés dans la région de Leleque que dans les années 1890, pour échapper aux campagnes militaires de l'Argentine et du Chili. Ces expéditions auraient donc été provoquées par ce «mélange explosif» entre les Mapuche et les Tehuelche ${ }^{16}$. Une fois de plus, selon un exercice d'attribution d'ethnicité qui déplore le métissage, les responsabilités historiques portent sur les indigènes, et la population d'origine européenne serait venue apporter une solution à des problèmes qu'ils ne furent pas capables de résoudre eux-mêmes.

Ces affirmations ne sont pas tenables très longtemps et elles ont toutes été réfutées par plusieurs chercheurs à différentes occasions. Le rôle des fonctionnaires coloniaux et des agents de l'état dans l'imposition d'une grille ethnique a largement été démontré. Certains auteurs ont directement mis en cause la construction de ces «panoramas » ou "cartes ethnologiques ", constitutifs des nations modernes et dont ont été friands les anthropologues de la première moitié $\mathrm{du} \mathrm{xx}^{\mathrm{e}}$ siècle. Sans les outils épistémologiques pour reconnaître la dynamique et la manipulation des identités, ces pratiques ethnologiques ont prolongé les effets du colonialisme en imposant des grilles ethniques artificielles qui ont abouti à une ébauche simpliste d'un "paysage culturel fait de segments» (Boccara 2001).

Que les ethnologues traditionalistes ne fassent plus autorité dans le cadre académique ne les empêche pas de conserver leur crédibilité quand il s'agit de vulgarisation, dans la mesure où leurs postulats sont compatibles avec les préjugés et les résistances à l'acceptation de l'altérité. Il faut aussi préciser que ces arguments ont été utilisés lors de l'inauguration de la nouvelle exposition du musée Leleque pour dénier aux communautés mapuche le droit à réclamer les terres de la région: leurs ancêtres ne seraient pas originaires de la Patagonie. En même temps, le musée propose l'exaltation iconographique des Tehuelche, ce peuple qui aurait été déplacé par les migrations mapuche de la fin du xix ${ }^{e}$ siècle. Casamiquela considère que les Tehuelche se sont éteints au milieu $\mathrm{du} \mathrm{xx}^{\mathrm{e}}$ siècle pour n'avoir pu conserver ni la pureté de leurs gènes ni leurs attributs culturels, pour avoir perdu leurs traditions et leurs langues günün a iajüch et aonik'o ais avec les dernières générations.

De cette manière est niée la légitimité des indigènes de Patagonie à se considérer comme tels, du fait de ne pas être «culturellement purs ». Le caractère d'étranger du Mapuche est mis en avant, ainsi que son manque de profondeur historique en Patagonie. Les revendications politiques et sociales adressées à l'État et aux grands propriétaires fonciers sont repoussées, tout autant que l'émergence de nouvelles identités comme la communauté autodéfinie mapuche-tehuelche, issue $\mathrm{du}$ processus politique de récupération de la fierté ethnique à la suite du cinquième centenaire de la conquête de l'Amérique. En ce sens, le révisionnisme ethnologique du musée Leleque cautionne les politiques de classe. En assumant la représentation et la préservation du patrimoine d'un peuple qu'il considère comme disparu, l'opération de mise sous silence fonctionne parfaitement, puisqu'il n'est plus d'héritiers pour revendiquer quelque droit que ce soit.

Face à l'«envahisseur" mapuche venu de l'autre côté des Andes, le revirement idéologique du musée Leleque a érigé le panthéon du bon Tehuelche, «agonisant » et argentin. La négation des sujets et des identités sociales et leur remplacement par d'autres culminèrent avec l'utilisation d'adjectifs dépréciatifs et paternalistes. Ainsi une dichotomie artificielle s'est mise en place au cours $\mathrm{du} \mathrm{xx}^{\mathrm{e}}$ siècle entre les descendants des 
Mapuche «belliqueux » et «les descendants de Tehuelche ». Ces derniers «jouèrent un rôle très secondaire, passif » et conservèrent « de leurs ancêtres paléolithiques l'habitude de la chasse nomade du gros gibier, le patriarcat, l'amour de la liberté et leur vision naive de l'univers et des hommes ", nous renseigne le prospectus du musée Leleque disponible sur le site Internet de Benetton-Patagonie.

Une telle description des hommes primitifs, le mépris pour le changement et l'imposition de typologies rigides permettent d'oublier que, depuis le début du $\mathrm{XIX}^{\mathrm{e}}$ siècle, «Mapuche» est le nom générique de l'altérité, un synonyme d'«indigène» par opposition à "Européen ", désigne les "gens de la terre " ou encore constitue un réceptacle de la diversité des identités régionales de la Pampa, du nord et du centre de la Patagonie et de l'Araucanie. Selon les spécialistes, le processus de construction d'une forme générique d'identité remonte au moins au XVII ${ }^{\mathrm{e}}$ siècle. Au début du XIX $\mathrm{X}^{\mathrm{e}}$ siècle, celui-ci était consolidé dans la Pampa et au nord de la Patagonie. Quelques années avant les conquêtes militaires, le métissage et l'hybridation culturelle caractérisaient la province actuelle du Chubut, conséquence de différents facteurs comme les affrontements pour le territoire, la capture de cautivos ou prisonniers, le jeu des alliances, le commerce de moyenne et de longue distance, les échanges de femmes ou les mariages interethniques à visée politique.

41 Les réseaux de sociabilité et de parenté, les marchés et la complexité des relations déterminées par le contact avec les chrétiens des frontières hispano-créoles finirent par configurer une identité indigène commune, de portée supra-régionale, qui se donne à voir dans les langues, les noms des individus, des familles, et des lieux de Patagonie et de la Pampa. Les ethnologues traditionnels qui ont précédé Rodolfo Casamiquela, malgré leur ethnocentrisme tenace, comprirent clairement la dimension de ces processus historiques. Parmi eux se détache Tomás Harrington, un maître d'école qui a partagé plusieurs années la vie des communautés indigènes du Chubut au tout début du xxe siècle. En faisant référence aux Mapuche et aux groupes de Tehuelche du nord et du sud, et après avoir confronté les noms ethniques attribués extérieurement avec ceux que les indigènes se donnaient eux-mêmes, Harrington commentait en 1942 que les «[...] Araucano, Günüa Küne et Aóeni Kenk étaient très mélangés entre eux pendant la seconde moitié du XIXe siècle ». Il ajoutait que, de ce fait, « [...] il n’y avait pas de Günüa Küne ni d'Aóeni Kenk pur » et que le "processus de dissolution » de la spécificité de chacune de ces ethnies «[...] est opératoire depuis fort longtemps avant la seconde moitié du XIX siècle ${ }^{17}$ ».

Comme l'a montré Jean-Loup Amselle, le colonialisme a favorisé la formation de groupes ethniques en Afrique en instituant des identités par l'action des missionnaires, des fonctionnaires et des ethnographes qui, dans de nombreux cas, ne constituaient qu'une seule et même personne. Réappropriées a posteriori par les acteurs locaux, les identités ethniques africaines, ou encore celles qui s'articulent dans les anciennes Républiques soviétiques, existent malgré leur création externe et imaginaire (Amselle 1998 : XIII-XV). Une expérience similaire s'est déroulée avec les Tehuelche et les Mapuche. Si leur défaite face aux États-nations argentin et chilien accéléra la synthèse, les indigènes de Patagonie et transandins peuvent légitimement se percevoir eux-mêmes comme une totalité depuis la fin du XIX ${ }^{e}$ siècle.

Le changement de cap du musée Leleque doit être compris à l'aune de ces questions. Si quelques photographies de la première exposition ont été maintenues dans la seconde, leur signification a glissé vers un nouveau récit qui met l'accent sur la «tehuelchisation » 
de la Patagonie, conformément au paradigme diffusionniste de l'école des kulturkreises et ses tendances à supprimer l'historicité des processus de construction de l'ethnicité, désormais à l'œuvre dans cette proposition muséographique de divulguer la «taxinomie des ethnies protohistoriques ${ }^{18}$ ".

En caractérisant la société patagonique contemporaine, à l'intérieur de laquelle «la culture de l'ancien peuple tehuelche arrivait à sa phase finale ", le nouveau récit omet ou, plus exactement, déplace la reconnaissance des conflits et des tensions sociales. Le devenir qui conduit à « la société sédentaire » non seulement retombe dans la confusion évolutionniste entre les pratiques saisonnières des indigènes et un nomadisme présenté comme arriéré, mais aussi supprime le rôle des grandes estancias comme unité de production de marchandises, d'ordre et de subjectivité. Dans ce contexte, le nouveau prospectus distribué aux visiteurs signale que «les Tehuelche découvrirent les biens à double tranchant de la civilisation ». À ce titre, «les bijoux, les vêtements, les produits alimentaires, les armes, l'alcool et les chevaux » sont interprétés comme des éléments de corruption culturelle. Désormais, le réflexe d'intolérance porte également sur l'immigration d'origine européenne et syrio-libanaise, par la récupération anachronique des diagnostics les plus sombres et les plus xénophobes du positivisme proposés au début $\mathrm{du} \mathrm{xx}^{\mathrm{e}}$ siècle, quand les élites argentines se rendirent compte que cet appel lancé aux populations d'outre-mer ne satisfaisait pas immédiatement leurs attentes. énoncés au XXI ${ }^{e}$ siècle, les propos relatifs aux « effets positifs et négatifs » de l'immigration populaire et à « l'occupation massive sans aucun critère de sélection » déplorent de manière tardive les conséquences de l'ethnocide, en lui attribuant des explications banales, en volant au secours paternaliste d'un indigène primitif, support idéal d'une identité nationale argentine en crise, qui s'interroge encore sur ses mythes fondateurs.

Manifestation indigène face au musée Leleque, 12 mai 2000.

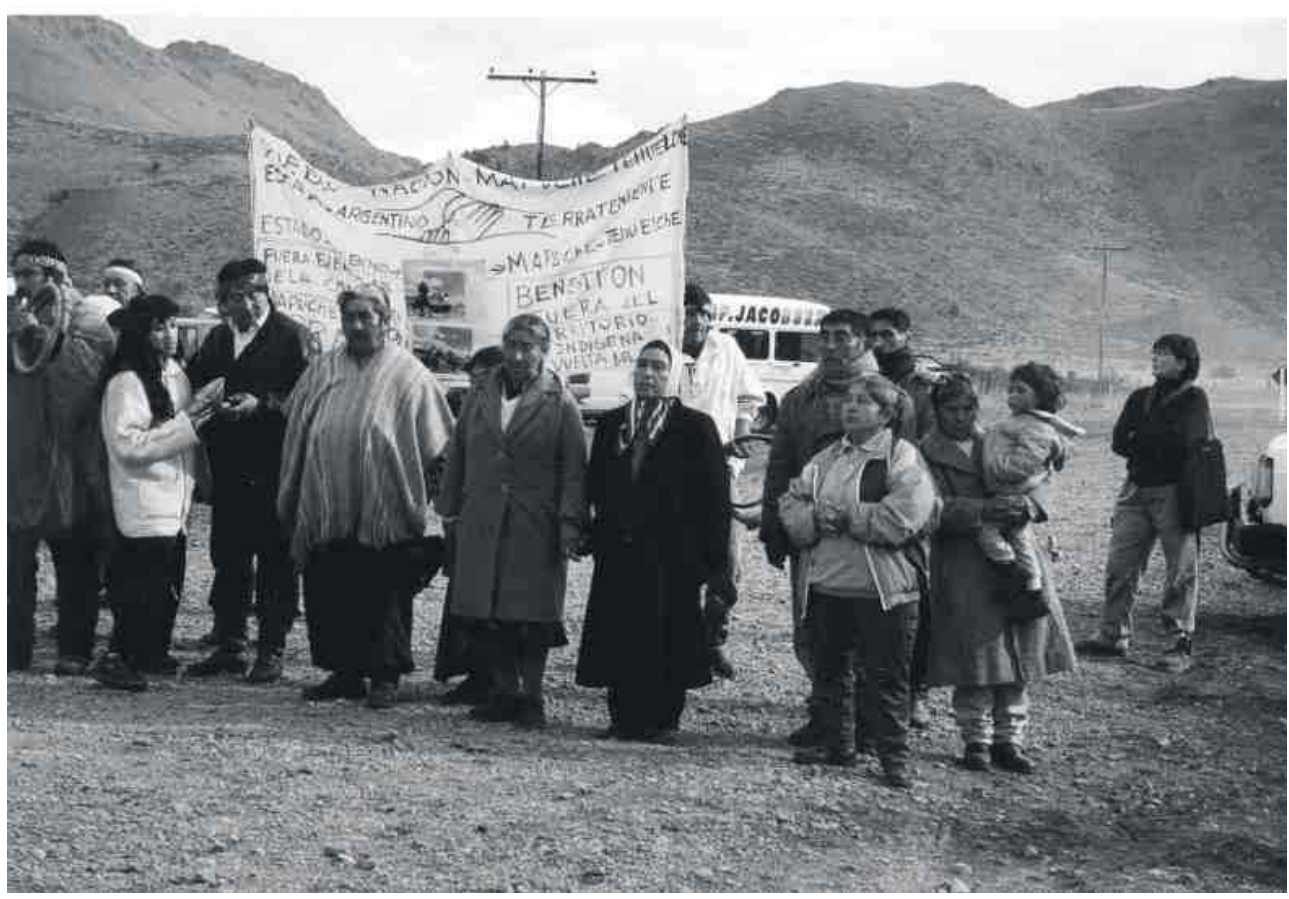

(c) Renata Biernat 
45 le caractère idéologique de la transformation des indigènes en "pièces de musée ", de
leur «muséification » en tant qu'ancêtres vivants de la nationalité. De telles pratiques ont vu le jour dans les années 1880 au musée des Sciences naturelles de La Plata. Son fondateur, Francisco Pascasio Moreno, fut un des scientifiques et explorateurs de la Patagonie à l'origine de l'imposition d'un ordre cognitif et d'un système de valeurs national fondés sur les fossiles et les pointes de flèches, une datation éloignée étant supposée consolider l'État-nation argentin ${ }^{19}$.

46

l'enceinte du musée de La Plata, parfois pendant toute une vie, à mi-chemin entre les pratiques de l'hospice, la satisfaction de la curiosité zoologique et l'intérêt personnel pour protéger une série de chefs et de familles avec qui il avait tissé des liens sentimentaux depuis ses expéditions de 1875 et 1880 . Mónica Quijada a bien montré que si l'ambiguïté prévalait en ce qui concerne les motivations d'un Moreno, qui alla jusqu'à faire sortir un groupe d'indigènes de prison, les raisons avancées pour justifier leur transfert aux autorités, ainsi que ses propres convictions renvoyaient à des arguments scientifiques. Il voyait dans le fait de leur offrir un logement au musée la possibilité de réaliser, à partir de ces «expositions vivantes", des études anthropologiques, physiques et culturelles d'une race en voie d'extinction.

L'un des caciques concernés était Foyel. Un portrait photographique de ce dernier, réalisé par un assistant de Moreno, est aujourd'hui exposé en grand format au musée Leleque. Cette conception de Foyel et de ses compagnons de réclusion comme "pièces de musée " - idée qui se prolonge immédiatement dans la conservation des restes mortuaires de quelques-uns d'entre eux dans les collections du musée de La Plata - est donc reprise par la muséographie actuelle de Leleque. Mais le nouveau scénario a tout de même modifié les conceptions qui avaient prévalu à l'édification, il y a plus d'un siècle, du musée de La Plata, et selon lesquelles le musée est un pilier scientifique de la nationalité, et l'ancien Tehuelche, l'ancêtre de la patrie. Il n'est plus question aujourd'hui de recevoir des indigènes dans les salons, les laboratoires ou les jardins. Leur seule présence viendrait interrompre l'histoire, comme l'a montré l'épisode de l'inauguration. Il n'est plus question que de leur exclusion et de leur essentialisation comme vestige exotique du passé pour le récit. Il s'agit de parler d'eux mais sans eux, de les représenter sans les présenter. "Nous ne sommes pas venus vous écouter », lança une femme de l'assistance aux manifestants des communautés indigènes qui interrompirent la présentation d'un livre récent de Casamiquela à Esquel, traitant précisément de la reconstruction rétrospective des anciens lignages indigènes (2004). 


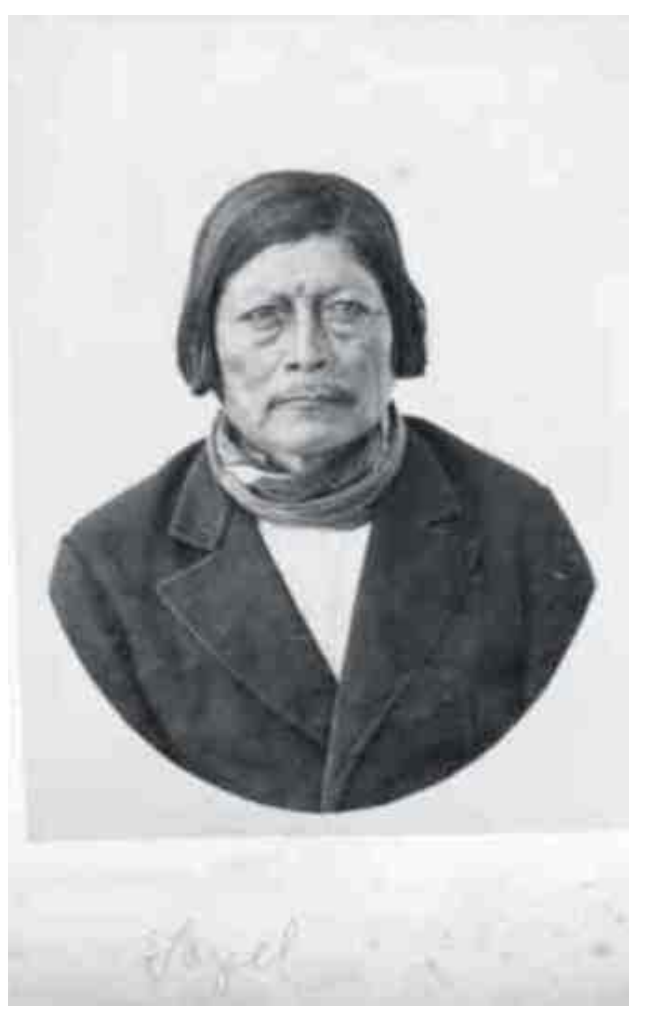

Contrairement au cas d'un Moreno, fonctionnaire de l'État, qui racontait la genèse de la nation en 1880 à partir de collections de pierres et d'êtres primitifs, il s'agit maintenant de substituer à l'État une entreprise privée qui se charge d'articuler les liens symboliques et d'instituer des subjectivités. Finie l'imposition d'identité par le fonctionnaire colonial, le voyageur positiviste ou l'ethnologue diffusionniste: le musée Leleque articule une opération moins subtile mais non moins complexe, qui récupère et combine ces anciennes pratiques.

Comme l'a indiqué Ignacio Lewkowicz (2004), l'État-nation devenu État-administratif se contente d'observer à distance, sans intervenir. Ce sont les agents actifs du marché qui, face au retrait de l'État souverain, s'occupent de configurer et de destituer les identités. Avec quelques contradictions - un seul Tehuelche emblématique devient l'unique représentant d'une diversité multicolore pourtant partout affichée -, l'intégration dans le musée Leleque de l'iconographie pluriethnique des boutiques de Benetton illustre les réflexions de Serge Gruzinski (1999) à propos du multiculturalisme comme illusion de diversité qui masque la différence dans le contexte de la globalisation, une "pluralité imaginaire » qui «défend des spécificités irréductibles » à un quelconque processus de métissage ou d'hybridation.

\section{Le bilan de l'expérience}

Le changement d'exposition opéré au musée Leleque ne doit pas masquer le fait que, quelle que soit la conception qui la fonde, l'exposition muséographique est le format narratif dans lequel s'insinuent fréquemment les zones d'ombre du discours historiographique : la fonction évocatrice des objets, des photographies, de la mise en 
scène ou de la fiction, les difficultés pour concilier histoire et mémoire et l'abus de la représentation par le montage (Giuderi 1992).

Ni la reconnaissance centrale du conflit social, ni la dénonciation du pillage et du génocide des peuples natifs qui caractérisaient la première exposition n'ont été suffisants. La correction politique et épistémologique a été phagocytée par le lieu d'énonciation du récit et par les directives tacites qui obligèrent à négocier les pratiques et les temporalités. Si la présence d'une cacica de la province voisine légitimait la cérémonie inaugurale, la protestation simultanée des groupes indigènes de l'ouest du Chubut a montré que l'appel lancé pour son organisation ne fut pas assez large. S'il l'avait été, nous aurions du comprendre depuis le début que, dans les conditions issues d'un patronage par le groupe Benetton, le musée Leleque était irréalisable. Malgré la richesse des témoignages recueillis pendant des dizaines d'années par les chercheurs de l'équipe, leur mise en scène aboutit à une représentation où les sujets étaient à peine interpellés. Pour parler comme le fait Franck Beuvier (2003: 122) à propos de l'exposition Le Musée cannibale, les vicissitudes du musée Leleque ont montré «[...] un traitement de l'objet ethnographique qui témoigne davantage d'une construction fantasmatique de l'Autre que de sa réelle connaissance ».

La marque Benetton a primé sur les intentions et les discours. Cette situation a été rendue possible par les vides du cadre légal argentin de régulation des initiatives qui combinent la participation d'organismes publics et celle d'entreprises privées dans la gestion du patrimoine archéologique, historique ou ethnographique.

53 Une fois la nation rendue opaque et le musée devenu relique de la patrie ou de la région épuisée, la Patagonie se redéfinit comme un lieu à territorialiser, un dernier espace exotique et incommensurable, ouvert à toutes les fictions des nouvelles découvertes. Tel est le regard proposé par Carlo Benetton dans Patagonia 13000 años de historia, livre qui prétend faire la synthèse des différentes conceptions historiographiques et anthropologiques des personnes ayant participé à l'expérience (Boschín et Casamiquela 2002) et qui, de ce fait, ne peut échapper aux contradictions. Dans l'introduction, l'entrepreneur a exprimé sa fascination pour la nature vierge et la beauté primitive d'un lieu idéal situé aux confins du monde. Le format United Colors of Benetton s'est autonomisé au point de devenir irréductible aux différentes propositions que nous avons formulées pour le premier projet et le premier montage. La nouvelle exposition a limé ces contradictions et a fait correspondre l'idéologie et les intentions de l'entrepreneur avec les travaux des responsables actuels de l'histoire racontée par le musée.

Dans le cadre de la conférence tenue à Rome en 2004 pour négocier la restitution des terres, les représentants des communautés indigènes réclamèrent à Benetton la "restitution de tous les éléments de leur culture qui sont exposés au musée Leleque ». Mauro Millán affirma à cette occasion : « Non seulement nous y croyons, mais nous nous fions également à des études d'anthropologues et d'historiens reconnus qui affirment que ce musée instaure l'idée que les peuples natifs ont disparu, qu'ils appartiennent au passé, que nous n'existons ni culturellement, ni physiquement. Tous les musées proposent un message idéologique et ce message est d'autant plus fort si l'on considère que le musée appartient à Benetton ${ }^{20}$. " Je souscris entièrement à ce point de vue, et ce à partir de la critique de ma participation à cette expérience aussi enrichissante que complexe. 


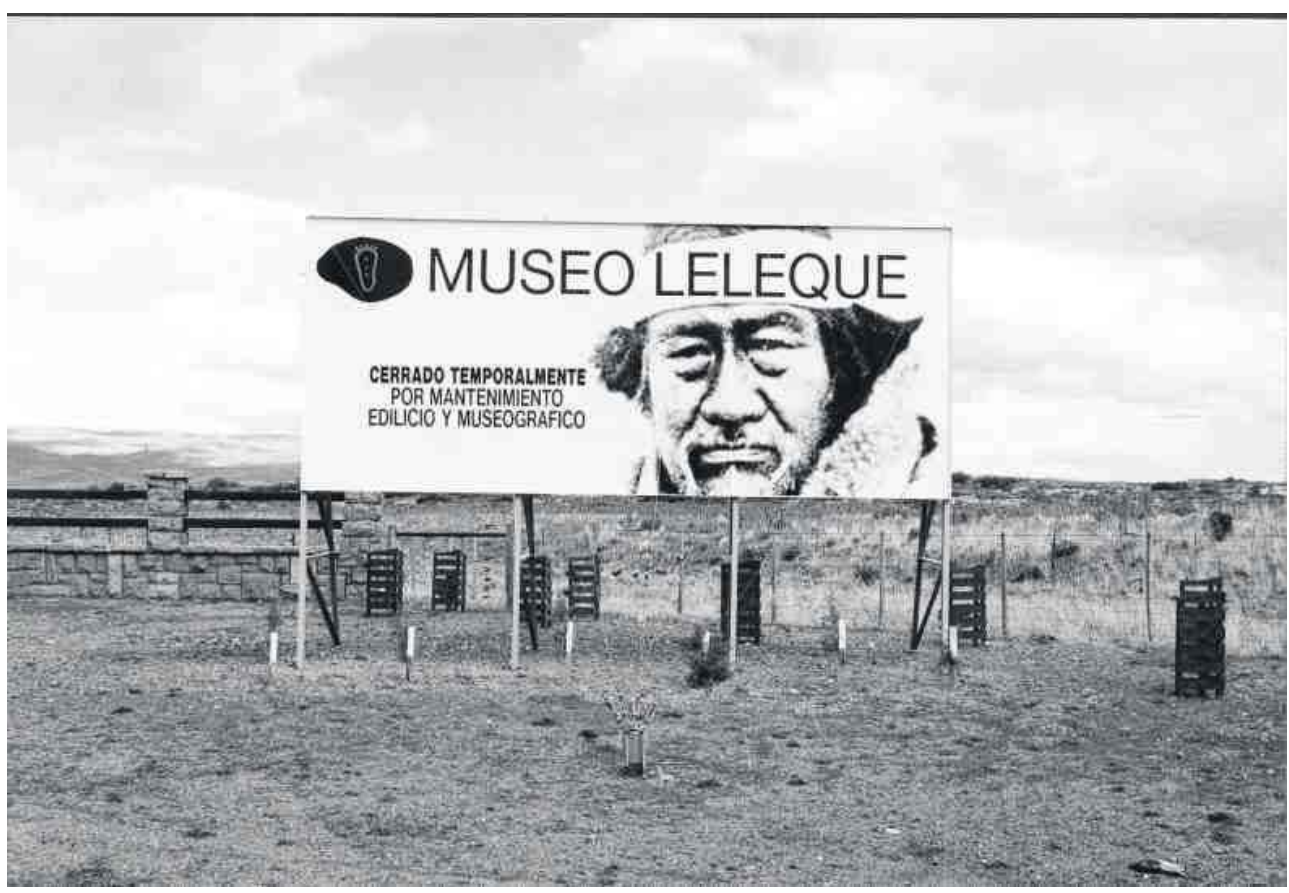

\section{BIBLIOGRAPHIE}

ALVAREZ, Gregorio

1972-1988 Neuquén. Historia, geografía, toponimia. Buenos Aires, Imprenta del Congreso de la Nación.

AMSELLE, Jean-Loup

1998 « Preface to the English-Language Edition. The Anthropologist Faces the "Hardening" of Identities », in Mestizo Logics : Anthropology of Identity in Africa and Elsewhere. Stanford, Stanford University Press.

AMSELLE, Jean-Loup, DUPUIS, Annie, éds.

1999 « Prélever, exhiber. La mise en musées », Cahiers d'études africaines XXXIX (3-4) : 155-156.

BECHIS, Martha

1992 « Instrumentos para el estudio de las relaciones interétnicas en el período formativo y de consolidación de estados nacionales ", in Cecilia Hidalgo et Liliana Tamagno, éd., Etnicidad e identidad. Buenos Aires, CEAL : 82-107.

BENJAMIN, Walter

1996 (1931) « Petite histoire de la photographie », Études photographiques 1, novembre, trad. A. Gunthert : 6-39. 
BEUVIER, Franck

2003 « Une muséologie critique des objets exotiques. Autour de l'exposition Le Musée cannibale », Gradhiva $33: 111-124$.

BOCCARA, Guillaume

1998 Guerre et ethnogenèse mapuche dans le Chili colonial. L'Invention du soi. Paris, L'Harmattan.

2001 « Mundos nuevos en la frontera del Nuevo Mundo », Nuevo Mundo, mundos nuevos 1 (http:// nuevomundo.revues.org).

Boschín, María T., CASAMIQUELA, Rodolfo, éd.

2002 Patagonia 13000 años de historia. Buenos Aires, Museo Leleque, Emecé.

CASAMIQUELA, Rodolfo

1965 Rectificaciones y ratificaciones. Hacia una interpretación definitiva del panorama etnológico de la Patagonia y área septentrional adyacente. Bahía Blanca, Cuadernos del Sur, Instituto de Humanidades de la Universidad Nacional del Sur.

1969 Un nuevo panorama etnológico del área pan-pampeana y patagónica adyacente. Pruebas etnohistóricas de la filiación tehuelche septentrional de los Querandíes. Santiago du Chili, Museo Nacional de Historia Natural.

1985 Bosquejo de una etnología de la provincia de Río Negro. Viedma, Fundación Ameghino.

1995 Bosquejo de una etnología de la Provincia del Neuquén. Buenos Aires, Ediciones La Guillotina.

2004 El linaje de los Yanquetruz. Confirmación genealógica de la presencia - en época histórica - del sustrato pantehuelche en el área pampeana. Trelew, Fundación Ameghino.

CERTEAU, Michel De

1978 L'Écriture de l'histoire. Paris, Gallimard.

cox, Guillermo

1863 Viaje en las rejiones septentrionales de la Patagonia, 1862-1863. Santiago du Chili, Imprenta Nacional.

DE AGostinI, Alberto

1945 Andes patagónicos. Viaje de exploración a la cordillera patagónica austral. Buenos Aires.

EVANS, Jessica

1999 « Introduction : Nation and Representation », in David Boswell et Jessica Evans, éds., Representing the Nation : A Reader. Histories, heritage and museums. Londres-New York, Routledge. GIUDERI, Remo

1992 Chronique du neutre et de l'auréole. Sur le musée et les fétiches. Paris, La Différence.

GRUZINSKI, Serge

1999 La Pensée métisse. Paris, Fayard.

HARRINGTON, Tomás

1946 « Contribución al estudio del indio Gününa Küne », Revista del Museo de La Plata, Sección Antropología, II : 237-275.

LEWKOWICZ, Ignacio 
2004 Pensar sin Estado. La Subjetividad en la era de la fluidez. Buenos Aires, Paidós.

MANDRINI, Raúl, ORTELLI, Sara

1992 Volver al país de los Araucanos. Buenos Aires, Sudamericana.

1995 «Repensando los viejos problemas : observaciones sobre la araucanización de las pampas », Runa - Archivo para las Ciencias del Hombre XXII : 135-150.

NABEL, Paulina

2000 « Museo Leleque », Ciencia Hoy $58:$ 58-60.

PODGORNY, Irina

1995 « De razón a Facultad : ideas acerca de las funciones del Museo de La Plata en el período 1890-1918 », Runa - Archivo para las Ciencias del Hombre XXII : 89-104.

QUIJADA, Mónica

1998 « Ancestros, ciudadanos, piezas de museo. Francisco P. Moreno y la articulación del indígena en la construcción nacional argentina », Estudios Interdisciplinarios de América Latina y el Caribe IX : 2.

VEZUB, Julio

2002 Indios y soldados. Las Fotografías de Carlos Encina y Edgardo Moreno durante la « Conquista del Desierto ». Buenos Aires, El Elefante Blanco.

VIGNATI, MilcíadesAlejo

1942 «Iconografía Aborigen, I. Los caciques Sayeweke, Inakayal y Foyel y sus allegados », Revista del Museo de la Plata (nouvelle série), Sección Antropología II : 13-48.

WALTHER, Juan Carlos

1947 La Conquista del desierto. Buenos Aires, Círculo Militar.

\section{NOTES}

1. La Compañía de Tierras Sud Argentino S.A., qui appartient au groupe Benetton, a participé à hauteur de 30000 pesos par an au financement des activités de l'équipe scientifique. Avant la dévaluation du peso argentin de décembre 2001, cela correspondait à 30000 dollars. Depuis lors, il faut diviser par trois cette valeur en monnaie américaine.

2. Les photographies historiques exposées au musée Leleque ont été extraites pour la plupart de l'album Encina, Moreno y Cía. Vistas fotográficas del Territorio Nacional del Limay y Neuquén (1883) et d'un article de Milcíades Alejo Vignati (1942). Les photographies du missionnaire salésien ont été publiées dans différents ouvrages, en particulier dans l'édition espagnole de son propre livre (De Agostini 1945).

3. Élise Dubuc, citée par Franck Beuvier (2003: 122).

4. Ce camélidé (Lama guanicoe) d'environ $100 \mathrm{~kg}$ constituait la principale ressource en viande et en cuir des chasseurs de la Patagonie.

5. Sur l'aura des photographies, voir Walter Benjamin (1996).

6. Sous le titre Prélever, exhiber. La mise en musées, ce volume des Cahiers d'études africaines (1999) est entièrement consacré aux problèmes du monde africain et des musées, notamment à la place de l'Afrique dans les musées occidentaux et la muséologie actuelle. L'expansion territoriale argentine et chilienne de la fin du XIX ${ }^{\mathrm{e}}$ siècle peut aussi se penser dans les termes d'un 
colonialisme qui produit des objets ethnographiques en les intégrant à des collections, comme le propose Annie Dupuis pour le cas africain dans la présentation de ce numéro des Cahiers. L'analyse d'Anne Doquet, «Les masques dogon : de l'objet au musée de l'Homme à l'homme objet de musée ", à propos du parcours des masques et de la mise en scène muséographique d'un village malien comme théâtre pour les danses masquées, offre un certain parallèle avec la prétention de développer un musée "vivant » autour des indigènes de Patagonie, organisé par une série de montages évocateurs, lesquels se révèlent incapables de rendre compte des lignes de force qui structurent une expression culturelle. Pour l'approfondissement des logiques coercitives et le désintérêt à l'égard de toute subjectivité non occidentale, voir, dans le même volume, l'article d'Ibéa Atondi, « La violence muséale : aux origines d'un discours ambigu ».

7. Juana Céliz, « Reportaje a Moira Millán. Cuestión de identidad», Hecho en Bs. As. Saliendo de la calle 38. Buenos Aires, 2003.

8. Témoignage recueilli par Paulina Nabel (2000: 59) le jour de l'inauguration.

9. Eduardo Videla, « Reclamo por tierras con buen rating », Página 12. Buenos Aires, 13 mai 2000.

10. Luis Frontera, « La Patagonia cuenta su historia », Revista Nueva. Buenos Aires, 23 juillet 2000.

11. Ibid.

12. Le livre de Juan Carlos Walther (1947) et l'œuvre de Gregorio Alvarez (1972-1988) sont les deux travaux les plus significatifs qui reproduisent, dans le registre de l'épique, quelques-unes des photographies de l'album des ingénieurs Encina et Moreno.

13. Procès $n^{\circ} 2740$ devant le Juzgado de Instrucción de la ville d'Esquel, folio 166, 2002.

14. La Nación. Buenos Aires, 9 novembre 2004.

15. En ce qui concerne cette problématisation de l'ethnicité et du contact en Patagonie, en Araucanie et dans la Pampa, voir, entre autres, Mandrini et Ortelli (1992 et 1995), Bechis (1992) et Boccara (1998).

16. Voir http://www.benetton.com. Conformément aux déclarations de Rodofo Casamiquela, «[...] les Mapuche arrivèrent en Patagonie avec la Conquista del Desierto entre 1890 et 1900. Rares sont les véritables Mapuche. L'expression mapuche en Argentine date des années 1960. Le Mapuche n'a pas de profondeur historique, seulement cent ans ici. [...] Les droits que les Mapuche réclament aujourd'hui sur la terre n'existent pas. [...] C'est de l'histoire. Ce n'est pas de la théorie. Aujourd'hui les choses changent politiquement. La question, c'est la terre. Du coup, les Mapuche du Chili disent qu'ils sont argentins et qu'il résident en Argentine depuis toujours. Mais cela n'intéresse pas l'historien, et les politiques et les avocats n'en savent rien ». Ces opinions synthétisent la pensée du directeur actuel du musée Leleque et sa façon de se poser en légataire d'une culture et en donneur d'identité : «[...] je ne viens pas parler des Mapuche mais présenter le monde tehuelche", conclut Casamiquela. Entretien réalisé par Estela Jorquera pour le quotidien Río Negro du 6 septembre 2004 publié sous le titre «Les véritables Mapuche sont très peu nombreux ».

17. Lettre citée par Vignati (1942:14). Voir également Harrington (1946).

18. Voir http://www.benetton.com.

19. Le cas historique du musée des Sciences naturelles de La Plata rentre parfaitement dans le cadre occidental qui veut que ce que l'on nomme un «musée ", inventé pendant la seconde moitié $\mathrm{du} \mathrm{XVIII}^{\mathrm{e}}$ siècle, célèbre l'unité de l'Etat-nation et matérialise une culture aux éléments nationaux (Evans 1999 : 6).

20. Déclarations au quotidien Jornada du 5 novembre 2004. 


\section{RÉSUMÉS}

Le groupe Benetton a financé la réalisation d'un musée d'histoire régionale, qui a ouvert ses portes en mai 2000 au milieu d'une grande estancia dont la société est propriétaire dans le nordouest de la Patagonie argentine. C'est à partir de l'expérience de l'un des membres de l'équipe d'anthropologues et d'historiens qui a élaboré les expositions du musée Leleque que sont analysées les utilisations et les interprétations de photographies de la fin du $\mathrm{XIX}^{\mathrm{e}}$ siècle représentant des indigènes alors définitivement écartés du contrôle du territoire de la Patagonie. L'article retrace les conflits de mémoire et ceux relatifs à la propriété de la terre qui se sont ouverts à cette occasion entre le groupe Benetton et les familles mapuche. Il s'interroge aussi sur les aspects éthiques de la participation de professionnels des sciences sociales à l'élaboration de récits historiques dans les conditions d'un parrainage privé.

The Benetton Group financed the setting up of a museum of regional history, which opened in May 2000, located on a large estancia owned by the group in northwestern Argentine Patagonia. Based on the experience of a member of the team of anthropologists and historians who planned the exhibitions housed in the Leleque museum, analysis is made of the use and interpretation of late 19th century photographs of natives with no outside territorial monitoring. The article takes a closer look at the conflicts of memory and over land ownership that the event gave rise to between the Benetton Group and Mapuche families, and also considers the ethical aspects of participation by social sciences professionals in the drawing up of historical accounts in a context of private patronage.

\section{INDEX}

Mots-clés : ethnicité, mapuche-tehuelche, muséologie, parrainage, Patagonie

Keywords : ethnicity, mapuche-tehuelche, museology, Patagonia, sponsorship

\section{AUTEURS}

\section{JULIO VEZUB}

Centro Nacional Patagónico - CONICET, Argentine, vezub@cenpat.edu.ar 